Article

\title{
Smart Collaborative Performance-Induced Parameter Identification Algorithms for Synchronous Reluctance Machine Magnetic Model
}

\author{
Linjie Ren ${ }^{1,2,3,4} \oplus$, Guobin Lin ${ }^{4}$, Yuanzhe Zhao ${ }^{1,2,3,4, * \mathbb{C}}$ and Zhiming Liao ${ }^{4, *}$ \\ 1 Key Laboratory of Road and Traffic Engineering of the State Ministry of Education, Shanghai 201804, China; \\ org0000h@tongii.edu.cn \\ 2 Key Laboratory of Rail Infrastructure Durability and System Safety, Tongji University, Shanghai 201804, China \\ 3 College of Transportation Engineering, Tongji University, Shanghai 201804, China \\ 4 National Maglev Transportation Engineering R\&D Center, Tongji University, Shanghai 201804, China; \\ linguobin@tongji.edu.cn \\ * Correspondence: zhaoyuanzhe@tongji.edu.cn (Y.Z.); liaozhiming@tongji.edu.cn (Z.L.)
}

check for

updates

Citation: Ren, L.; Lin, G.; Zhao, Y.;

Liao, Z. Smart Collaborative

Performance-Induced Parameter

Identification Algorithms for

Synchronous Reluctance Machine

Magnetic Model. Sustainability 2021

13, 4379. https://doi.org/10.3390/

su13084379

Academic Editors: Yang Song,

Hongrui Wang and Yongqiu Zhu

Received: 5 March 2021

Accepted: 2 April 2021

Published: 14 April 2021

Publisher's Note: MDPI stays neutral with regard to jurisdictional claims in published maps and institutional affiliations.

Copyright: (c) 2021 by the authors. Licensee MDPI, Basel, Switzerland. This article is an open access article distributed under the terms and conditions of the Creative Commons Attribution (CC BY) license (https:// creativecommons.org/licenses/by/ $4.0 /)$.

\begin{abstract}
In rail transit traction, due to the remarkable energy-saving and low-cost characteristics, synchronous reluctance motors (SynRM) may be a potential substitute for traditional AC motors. However, in the parameter extraction of SynRM nonlinear magnetic model, the accuracy and robustness of the metaheuristic algorithm is restricted by the excessive dependence on fitness evaluation. In this paper, a novel probability-driven smart collaborative performance (SCP) is defined to quantify the comprehensive contribution of candidate solution in current population. With the quantitative results of SCP as feedback in-formation, an algorithm updating mechanism with improved evolutionary quality is established. The allocation of computing resources induced by SCP achieves a good balance between exploration and exploitation. Comprehensive experiment results demonstrate better effectiveness of SCP-induced algorithms to the proposed synchronous reluctance machine magnetic model. Accuracy and robustness of the proposed algorithms are ranked first in the comparison result statistics with other well-known algorithms.
\end{abstract}

Keywords: rail transit traction; synchronous reluctance machine; nonlinear magnetic model; parameters identification; population evolution mechanism; synergistic optimization

\section{Introduction}

The intensification of the greenhouse effect and the nonrenewability of rare earth resources are severe challenges for induction motors (IM) and permanent magnet motors (PM) [1]. The Synchronous Reluctance Motor (SynRM) is the most promising alternative due to its high efficiency and structural robustness [2]. With increasing application of SynRM, novel modeling theories [3] that can closely represent its nonlinear magnetic characteristic have drawn considerable attention in its operation mechanism analysis and high-performance control [4]. Although many magnetic models have been developed based on various nonlinear mapping [5], the physical rationality and parameter accuracy of these models restrict the full utilization of motor performance. Similar to the actual network system [6], the co-energy balance between the $\mathrm{d}-\mathrm{q}$ axis is the key constraint of modeling. Consequently, a novel magnetic model is established under the constraints of the inherent characteristics. However, there are still some challenges for the proposed model parameter identification, such as local optimal problems caused by the mismatch of special parameters of the algorithm and the multimodality of the objective function.

The SynRM magnetic model is significant for its control and practical application. For many advanced control algorithms, the rationality and accuracy of the model are the foundation [7]. In addition, for intelligent algorithms [8,9] with low model dependence, accurate models help to improve the performance of intelligent control systems. SynRM 
modeling technology with both physical consistency and accuracy is a realistic demand and has practical significance. However, the accuracy of the model is still limited by the rationality of the parameter identification algorithm.

Existing research mainly focuses on two types of methods: deterministic and metaheuristic. In [10], least squares (LS) method has been used in the extraction of magnetic model parameters. Suffering from sensitivity to initial solution and dataset noise, the convergence of LS results is unsatisfied and uncertain [11]. As an alternative, metaheuristic algorithms are employed to identify the parameters of various motor models. In [12], a genetic algorithm (GA) was proposed for estimating the parameters of a saturation model using experimental test data under three working conditions. In [13], harmony search (HS) was utilized to identify parameters of a rational function-based inductance model. In [14], two improved particle swarm optimization algorithms (PSO)- based strategies (dynamic PSO and chaos PSO algorithms) are proposed to estimate the parameters of the induction motor model. The direct current, no-load and locked-rotor test results are used for parameter estimation. Other algorithm variants such as self-adaptive differential evolution algorithm (SHDE) [15], dynamic encoding algorithm searches (DEAS) [16], fast parallel co-evolutionary PSO [17] and GA-assisted PSO algorithm [18] are employed to identify the parameters of the motor model. These metaheuristic algorithms have achieved satisfied results in parameter identification of the motor model. However, the multimodality of the problem places more stringent requirements on traditional metaheuristic algorithms.

The distinguishing feature of metaheuristic algorithms is to construct the optimal population update mechanism. It is a key issue to fully exploit fitness information while maintaining diversity for designing an efficient algorithm. Its essence is the balance of computing resources under multiple dimensions and multiple influencing factors [19]. To improve the population diversity, probability-based attempts have been made. In [20], the probability of fitness evaluation was used to establish more promising searching direction for maintaining diversity. A similar method was adopted to promote exploration ability of differential evolution (DE) in [21]. Through nondominated sorting procedure, diversity information was included in the selection probability, which generated the mutation of the differential evolution (DE) algorithm [22]. However, due to the lack of a unified theory of the collaborative utilization of fitness information and diversity information, traditional metaheuristic algorithms may have defects in solving complex problems. On one hand, searching direction is only guided by fitness, weakening the exploration ability. On the other hand, excessive use of diversity information will weaken exploitation ability. In recent years, smart collaborative (SC) theory has shown outstanding advancement in the modeling [23], resource balancing of complex problems with multiple dimensions and multiple influencing factors. Inspired by the SC theory, the performance of individuals can be modeled from the two dimensions of fitness and diversity through probability.

The parameter estimation of the proposed model is a multimodal problem. There always exists a certain degree of nonlinear noise in the flux linkage data obtained by the experimental method or the finite element analysis (FEA). The multimodality caused by nonlinear noise may make the traditional identification algorithm invalid. Improper specific control parameter selection of many metaheuristic algorithms often results in premature termination. Furthermore, as feedback information for evolution, fitness is over-relied on and diversity information is often ignored. As a result, it may not be possible to obtain the global optimal solution. Hence, developing a competitive metaheuristic algorithm to identify the proposed magnetic model parameters is still a challenging task.

Therefore, we propose a smart collaborative performance (SCP) to quantify the comprehensive performance of individuals in the population through probability. As feedback information, SCP is used to induce the construction of the evolutionary direction of the population. In this way, the fitness and diversity contribution of the candidate solution to current population is introduced in the evolution simultaneously. The candidates with better comprehensive performance are encouraged into new populations, improving the quality of solution in each generation. In addition, the optimal search path update process 
is adaptive without additional control parameters. Specifically, three SCP-induced algorithms are proposed to identify the parameters of the SynRM magnetic model accurately and reliably. To evaluate the effectiveness of the proposed algorithms, we compared them with other well-established algorithms on parameter identification problems of the SynRM magnetic model.

The main contributions of this paper can be summarized as follows:

- The SCP-induced algorithms are proposed based on quantified SCP. Interactions among candidate solutions are introduced into the optimal search path updating to promote the related searching ability. To the best of our knowledge, no precedent that employs SCP-induced algorithms to extract the parameters of the SynRM magnetic model has been published or reported publicly;

- As a simple and effective quantitative indicator, SCP is defined to estimate fitness and diversity of candidates uniformly. In addition, the SCP-induced population evolution mechanism is established to select promising candidate solutions in the current population evolution.

The rest of the paper is organized as follows. Section 2 presents the SynRM magnetic model, and the problem formulation of the model. The overview of SCP-induced algorithms is given in Section 3. Experimental results on the multiparameter SynRM magnetic model are shown and analyzed in Section 4. Finally, the conclusions are drawn in Section 5.

\section{Problem Formulation}

In this section, we propose a novel magnetic model of SynRM. Objective function involved in parameter identification of the propose model is also defined. The proposed model is established under the following hypothesis: (1) The iron loss has been omitted. (2) The stator flux linkages are only dependent on stator currents. (3) The effect of temperature on stator resistance is neglected.

\subsection{Modeling of SynRM Magnetic Behaviors}

For SynRM, the electromagnetic torque is generated by an anisotropic rotor with a magnetic barrier in the coupled magnetic field. The main rotor topologies are axial lamination (ALA) and transverse lamination (TLA) [24]. The ALA rotor has higher anisotropy. However, its iron loss and processing difficulty are greater than TLA. In general, the internal mechanism of the torque generated by the two rotor structures is the same. Due to the material properties of the rotor, there is inevitably a self-saturation effect in the $d$ and $q$-axis magnetic circuits. In addition, due to the sharing of magnetic circuits, there is a cross-coupling effect between the $d$ - and $q$-axis magnetic linkages. The nonlinear behavior mechanism of the two rotors is the same, and the following general modeling method can be used.

The SynRM magnetic model is characterized by the nonlinear relationships among stator currents $\left(\boldsymbol{i}_{s}\right)$ and flux linkages $\left(\boldsymbol{\psi}_{s}\right)$. The mapping relationship between stator currents and flux linkages in $d-q$ reference frame is expressed by

$$
\left\{\begin{array}{l}
\psi_{d}=\psi_{d}\left(i_{d}, i_{q}\right) \\
\psi_{q}=\psi_{q}\left(i_{d}, i_{q}\right)
\end{array}\right.
$$

where $i_{s}=\left(i_{d}, i_{q}\right)^{\mathrm{T}}, \psi_{s}=\left(\psi_{d}, \psi_{q}\right)^{\mathrm{T}}$ are stator currents and flux linkages, respectively. The subscripts $d$ and $q$ represent $d$ - and $q$-axis. These nonlinear parameters are determined by the process of electromechanical energy conversion in the machine. The electrical and mechanical systems of the machine are coupled by the magnetic energy $\left(W_{m}\right)$ transformation in the magnetic field. Under the assumption of conservative fields, the energy in the coupled field is conserved. The energy balance equation can be defined as

$$
\boldsymbol{i}_{s}^{T} \boldsymbol{u}_{s}=R_{s}\left\|\boldsymbol{i}_{s}\right\|^{2}+\frac{d W_{m}}{d t}+T_{e}\left(\boldsymbol{i}_{s}\right) \omega_{m}
$$


where $u_{\mathrm{s}}=\left(u_{d}, u_{q}\right)^{\mathrm{T}}, R_{\mathrm{s}}, T_{e}\left(i_{\mathrm{s}}\right), W_{m}, \omega_{m}$ are stator voltages, stator winding resistance, electromagnetic torque, magnetic energy and mechanical velocity of the motor, respectively. The energy conversion in coupled magnetic field is determined by the variation of $W_{m}$, which is caused by the change of $\psi_{s}$. For convenience, the derivative of $W_{m}$ can be equivalently expressed by the derivative of comagnetic energy $\left(W_{c}\right)$ as

$$
\frac{d W_{c}}{d t}=\psi_{d} \frac{d i_{d}}{d t}+\psi_{q} \frac{d i_{q}}{d t}
$$

Due to the conservation of energy, the result is independent of the integral path when $W_{c}$ in (3) is calculated by curvilinear integration. Thus, the reciprocity condition [5] that $W_{c}$ needs to satisfy can be presented as

$$
\frac{\partial^{2} W_{c}}{\partial i_{d} \partial i_{q}}=\frac{\partial^{2} W_{c}}{\partial i_{q} \partial i_{d}} \Leftrightarrow \frac{\partial \psi_{d}}{\partial i_{q}}=\frac{\partial \psi_{q}}{\partial i_{d}}
$$

Combine with (3) and (4), the change of stator currents makes the $W_{c}$ change, and at the same time energy exchange occurs between the $d$ and $q$ axes. This reveals the formation mechanism of nonlinear behavior of SynRM magnetic circuit. The (8) and (9) also indicated that $\psi_{\mathrm{s}}$ is irrotational and $W_{c}$ is the scalar potential of $\psi_{\mathrm{s}}$. The divergence of $\psi_{\mathrm{s}}$ can be obtained by Laplacian operation of $W_{c}$

$$
\nabla^{2} W_{c}=f\left(i_{d}, i_{q}\right)
$$

where $f\left(i_{d}, i_{q}\right)$ is an unknown function with stator current as the independent variable; (5) is the Poisson's equation of $W_{c}$. Obviously, the analytic solution of this equation cannot be obtained directly. As is well known, the general solution often contains Gaussian's function terms. Hence, the nonlinear function based on the Gaussian's function can be used to model $W_{c}$. The variation of $W_{c}$ can be modeled as

$$
\Delta W_{c}=k\left(e^{-W i_{d}^{2}}-1\right)\left(V_{1} i_{q}^{2}-i_{q} e^{-V i_{q}}+\kappa_{q} i_{q}-e^{-V i_{q}}\right)
$$

where $k, W, V_{1}, V, \kappa_{q}$ denote the unknown parameter. Combine (3) and (6), the change of $W_{c}$ with current can also be expressed by flux linkages as

$$
\left\{\begin{array}{l}
\psi_{d}\left(i_{d}, i_{q}\right)=\psi_{d}\left(i_{d}\right)-\frac{\partial \Delta W_{c}}{\partial i_{d}} \\
\psi_{q}\left(i_{d}, i_{q}\right)=\psi_{q}\left(i_{q}\right)-\frac{\partial \Delta W_{c}}{\partial i_{q}}
\end{array}\right.
$$

where $\psi_{d}\left(i_{d}\right), \psi_{q}\left(i_{d}\right)$ are the flux linkages under the effect of self-saturation alone. The second term on the right side of (7) represents the flux linkages under the effect of crosssaturation. Considering the constraints of (7) and reciprocity condition simultaneously, the nonlinear flux linkage model of SynRM can be defined as

$$
\left\{\begin{aligned}
\psi_{d}\left(i_{d}, i_{q}\right) & =\alpha_{d}\left(2-e^{-\gamma_{d} i_{d}}-e^{-\gamma_{d 1} i_{d}^{2}}\right)+\beta_{d} i_{d} \\
& -k\left(2 W i_{d} e^{-W i_{d}{ }^{2}}\right)\left(V_{1} i_{q}^{2}-i_{q} e^{-V i_{q}}+\kappa_{q} i_{q}-e^{-V i_{q}}\right) \\
\psi_{q}\left(i_{d}, i_{q}\right) & =\alpha_{q}\left(2-e^{-\gamma_{q} i_{q}}-e^{-\gamma_{q} i_{q}^{2}}\right)+\beta_{q} i_{q} \\
& -k\left(1-e^{-W i_{d}{ }^{2}}\right)\left(2 V_{1} i_{q}+V i_{q} e^{-V i_{q}}+\kappa_{q}\right) \\
\frac{\partial \psi_{d}\left(i_{d}, i_{q}\right)}{\partial i_{q}} & =\frac{\partial \psi_{q}\left(i_{d}, i_{q}\right)}{\partial i_{d}}=-k\left(2 W i_{d} e^{-W i_{d}{ }^{2}}\right)\left(2 V_{1} i_{q}+V i_{q} e^{-V i_{q}}+\kappa_{q}\right)
\end{aligned}\right.
$$

where $\{\alpha, \gamma, \beta\}$ are non-negative self-saturation coefficients for the $d$-, and $q$-axis, and $\{k$, $\left.W, V, V_{1}, \kappa_{q}\right\}$ are cross-saturation coefficients. The subscripts $d$ and $q$ represent $d$-axis and q-axis. 


\subsection{Objective Function}

Parameter identification of the model can be transformed into an optimization problem, which aims to minimize the difference between the reference data and simulated data obtained by estimated parameters. Noteworthily, the cross-saturation coefficient must be consistent due to the reciprocity condition. The error functions for the $k$ th flux linkages respect to $d$ - and $q$-axis, respectively, can be formulated as

$$
\begin{aligned}
& \left\{\begin{aligned}
& f_{k}^{d}\left(\psi_{r}^{d}, i_{r}^{d}, i_{r}^{q}, x_{d}\right)= \alpha_{d}\left(2-e^{-\gamma_{d} i_{r}^{d}}-e^{-\gamma_{d 1}\left(i_{r}^{d}\right)^{2}}\right)+\beta_{d} i_{r}^{d} \\
&-k\left(2 W i_{r}^{d} e^{-W\left(i_{r}^{d}\right)^{2}}\right)\left(V_{1}\left(i_{r}^{q}\right)^{2}-i_{r}^{q} e^{-V\left(i_{r}^{q}\right)^{2}}+\kappa_{q} i_{r}^{q}-e^{-V i_{r}^{q}}\right)-\psi_{r}^{d} \\
& x_{d}=\left\{\alpha_{d}, \gamma_{d}, \beta_{d}, k, W, V, V_{1}, \kappa_{d}\right\}
\end{aligned}\right. \\
& \left\{\begin{array}{c}
f_{k}^{q}\left(\psi_{r}^{q}, i_{r}^{d}, i_{r}^{q}, x_{q}\right)=\alpha_{q}\left(2-e^{-\gamma_{q} i_{r}^{q}}-e^{-\gamma_{q 1}\left(i_{r}^{q}\right)^{2}}\right)+\beta_{q} i_{r}^{q} \\
-k\left(1-e^{-W\left(i_{r}^{d}\right)^{2}}\right)\left(2 V i_{r}^{q}+V i_{r}^{q} e^{-V i_{r}^{q}}+\kappa_{q}\right)-\psi_{r}^{q} \\
x_{q}=\left\{\alpha_{q}, \gamma_{q}, \beta_{q}, k, W, V, V_{1}, \kappa_{q}\right\}
\end{array}\right.
\end{aligned}
$$

Hence, the objective functions defined by root mean square (RMSE) error can be obtained as follows:

$$
\begin{aligned}
& \operatorname{RMSE}_{d}\left(x_{d}\right)=\sqrt{\frac{1}{N} \sum_{k=1}^{N} f_{d}^{k}\left(\psi_{r}^{d}, i_{r}^{d}, i_{r}^{q}, x_{d}\right)^{2}} \\
& \operatorname{RMSE}_{q}\left(x_{q}\right)=\sqrt{\frac{1}{N} \sum_{k=1}^{N} f_{q}^{k}\left(\psi_{r}^{q}, i_{r}^{d}, i_{r}^{q}, x_{q}\right)^{2}}
\end{aligned}
$$

where $x$ is the decision variable, $N$ is the sample number, $\psi_{r}, i_{r}$ are stator flux linkages and currents in the collected FEA dataset. $x_{d}, x_{q}$ are the obtained parameter vectors, where $x_{d}=\left[x_{d 1}, x_{c}\right]$, and $x_{q}=\left[x_{q 1}, x_{c}\right] . x_{d 1}$ and $x_{q 1}$ are the unique parameters involved in $x_{d}$ and $x_{q}$, respectively, and $x_{c}=\left[k, W, V, \kappa_{d}, \kappa_{q}\right]$ is the common parameter subvector. Here, the subscripts $d$ and $q$ represent $d$ - and $q$-axis, respectively. $N$ is the number of reference data.

\section{SCP-Induced Parameter Identification Algorithms}

In this section, detail of proposed algorithms will be given. First, a new performance indicator named SCP is proposed, which is unified quantification of the fitness and diversity contributions of the candidate solutions in current population. We describe three improved Rao algorithms [25] induced by SCP.

\subsection{Smart Collaborative Performance}

In general, the performance of the candidate solution is affected from two dimensions, fitness associated with exploitation and diversity related to exploration. In evolution of population, the balance between exploitation and exploration is the key to optimal algorithm design. For the candidate, fitness and diversity information is generated under the influence of evolutionary mechanism. In other words, these two kinds of information can reflect the evolutionary preferences of the current population. Therefore, if a reasonable comprehensive performance quantification standard can be determined, the evolution direction can be influenced through the feedback of quantified indicator. In [21] and [26], fitness and diversity can be calculated by the objective function and the distance between the individual and the surrounding individual, respectively. However, it is impossible to evaluate the overall performance of the corresponding solution through a simple comparison, due to the different properties of the two functions. In addition, even if a comprehensive evaluation method based on these two functions can be found, to quantify the contribution of candidate solutions to the population is still an issue. Inspired by the 
collaborative modeling method in [27], an SCP evaluation indicator for the $k^{\text {th }}$ candidate solution $x_{k}$ is defined as

$$
P_{s}\left(x_{k}, N P\right)=P_{d}\left(x_{k}, N P\right) \cdot c_{k, s}+P_{f}\left(x_{k}, N P\right) \cdot\left(1-c_{k, s}\right)
$$

where, $P_{s}\left(x_{k}, N P\right), P_{f}\left(x_{k}, N P\right), P_{d}\left(x_{k}, N P\right)$, represent the SCP, fitness performance (FP) and diversity performance (DP) for the candidate expressed in probability, respectively. $c_{k, s}$ is a random number in the range [0,1], denoting smart collaborative coefficient (SCC). The overall performance of the candidate in the current population is evaluated by SCP. It can be clearly observed that the candidate with better SCP has a larger probability. FP, DP represent the fitness and diversity contributions of the candidate in the current population, respectively. The evolutionary process preference for exploration and exploitation maintenance can be quantified by SCC, which also decided the proportion of FP and DP in SCP evaluation. With the increase of SCC, the preference for diversity increases in SCP. On the contrary, the preference for fitness increases in SCP. Particularly, when $c_{k, s}=0$ or $c_{k, s}=1, \mathrm{SCP}$ degrades into FP or DP. In this situation, SCP only evaluates the performance of the candidate from the perspective of fitness or diversity contribution. If SCP is used as feedback, the performance of the candidate is only affected by exploitation or exploration preference of evolution. As the basis of SCP construction, the quantification process of FP and DP is as follows.

The objective function $\left(f\left(x_{k}\right)\right)$ is selected as the fitness function $\left(f_{\text {fitness }}\left(x_{k}\right)\right)$ for each candidate solution.

$$
f_{\text {fitness }}\left(\boldsymbol{x}_{k}\right)=f\left(\boldsymbol{x}_{k}\right)
$$

Then the candidates are sorted in ascending order of fitness in the population, and probability based on ranking is used to reflect the fitness performance. The ranking and FP of $x_{k}$ are presented in (18) and (19), respectively.

$$
\begin{gathered}
R_{\text {fitness }}\left(x_{k}, N\right)=N P+1-i_{k}, i_{k}=1,2, \ldots, N P \\
P_{k, f}\left(x_{k}, N P\right)=\left(\frac{R_{\text {fitness }}\left(x_{k}, N P\right)}{N P}\right)^{2}
\end{gathered}
$$

where $i_{k}$ represents the fitness ranking of $x_{k}$ in the population.

The diversity function based on Euclidean distance between candidates is defined as

$$
f_{\text {diversity }}\left(\boldsymbol{x}_{k}\right)=-\sum_{w=1}^{N P}\left\|\boldsymbol{x}_{k}-\boldsymbol{x}_{w}\right\|, w=1,2, \ldots, N P
$$

Similar to (15) and (16), the diversity ranking and DP are presented as follows.

$$
\begin{gathered}
R_{\text {diversity }}\left(x_{k}, N P\right)=N P+1-j_{k}, j_{k}=1,2, \ldots, N P \\
P_{k, d}\left(x_{k}, N P\right)=\left(\frac{R_{\text {diversity }}\left(x_{k}, N P\right)}{N P}\right)^{2}
\end{gathered}
$$

where $j_{k}$ represents the fitness ranking of $x_{k}$ in current population. In (16) and (19), better performance corresponds to larger probability. The overall contribution of the candidates to the population is quantified by the random combination of the probabilities through SCP. As a result, SCP has advantage of theory-driven.

\subsection{SCP-Induced Optimal Search Path Update Mechanism}

Based on the above calculated probability, evolutionary preferences can be quantified by SCP, and the evolution process is also affected by SCP. As feedback information, SCP only affects the optimal solution search direction update mechanisms. Hence, SCP is used 
to modify the three Rao algorithms [25]. Thus, the new update mechanism based on SCP can be defined as

$$
\begin{gathered}
x_{k, v}^{\prime}=x_{k, v}+r_{1, k, v}\left(x_{\text {best }, v}^{s}-x_{\text {worst }, v}^{s}\right) \\
x_{k, v}^{\prime}=x_{k, v}+r_{1, k, v}\left(x_{b e s t, v}^{s}-x_{\text {worst }, v}^{s}\right)+r_{2, k, v} \Delta x_{k l, v}^{s} \\
x_{k, v}^{\prime}=x_{k, v}+r_{1, k, v}\left(x_{b e s t, v}^{s}-\left|x_{w o r s t, v}^{s}\right|\right)+r_{2, k, v} \Delta x_{k l, v}^{s}
\end{gathered}
$$

where the superscript " $s$ " represents the candidate for quantitative selection based on SCP. Subscripts "best" and "worst" represent the candidates with the largest and smallest SCP in the current population. In the proposed update mechanisms, no additional parameters are introduced. Evolutionary direction is still close to the best candidate solution and away from the worst candidate solution. In addition, $\Delta x_{k l}$ represents the difference between randomly selected candidates, always greater than zero.

With the introduction of SCP, the evaluation of the merits of the candidates is not only related to their own fitness and diversity, but also related to the current population. In other words, if the FP and DP of the candidates have not changed in the previous search direction update, they will be recalculated before the search direction update. Then, the candidate with a larger $P_{s}$ is selected in the comparison. The process of calculating $\Delta x_{k l}^{s}$ through SCP comparison is proposed in Algorithm 1. If $x_{\text {best }}^{s}, x_{\text {worst }}^{s}$ and $\Delta x_{k l}^{s}$ are used as evolutionary guidance, evolutionary preferences will be affected by the inherent randomness of their SCP. This random effect is automatic and does not require additional control parameters, hence the update direction of the candidate solution is expected to be guided into new search regions.

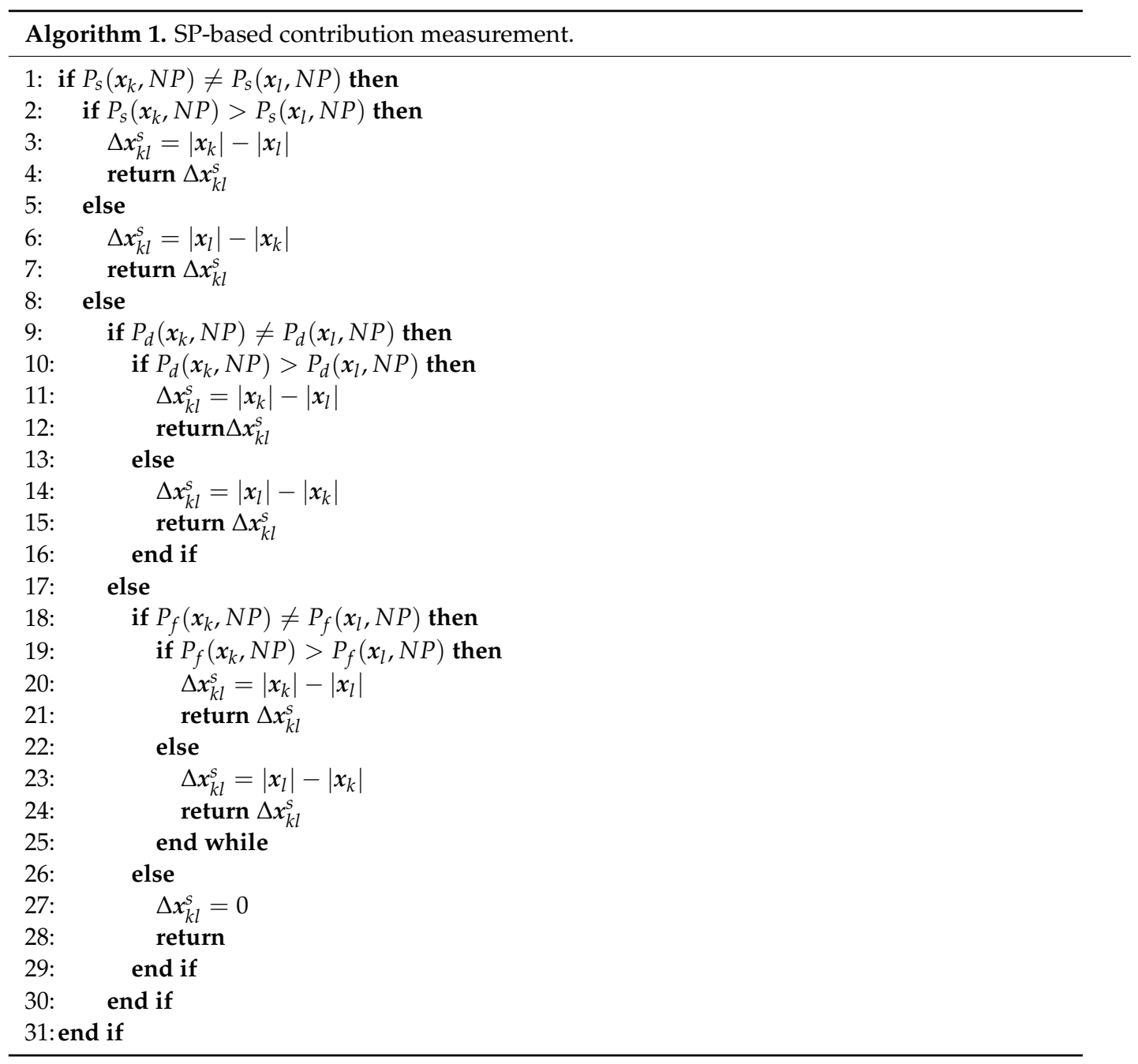




\subsection{SCP-Rs Framework}

For the proposed update mechanisms, due to the automatic intervention of evolutionary direction, the stagnation and premature convergence in the evolution process are avoided. To inherit the strong exploitation ability of the original Rao algorithms, a new framework combining the original update mechanisms and the modified update mechanism is proposed, named SCP-R algorithms. Corresponding to different update mechanisms, these algorithms are named as SCP-R1, SCP-R2 and SCP-R3. Algorithm 2 gives the pseudo code of SCP-R algorithms, and Figure 1 provides the flow chart.

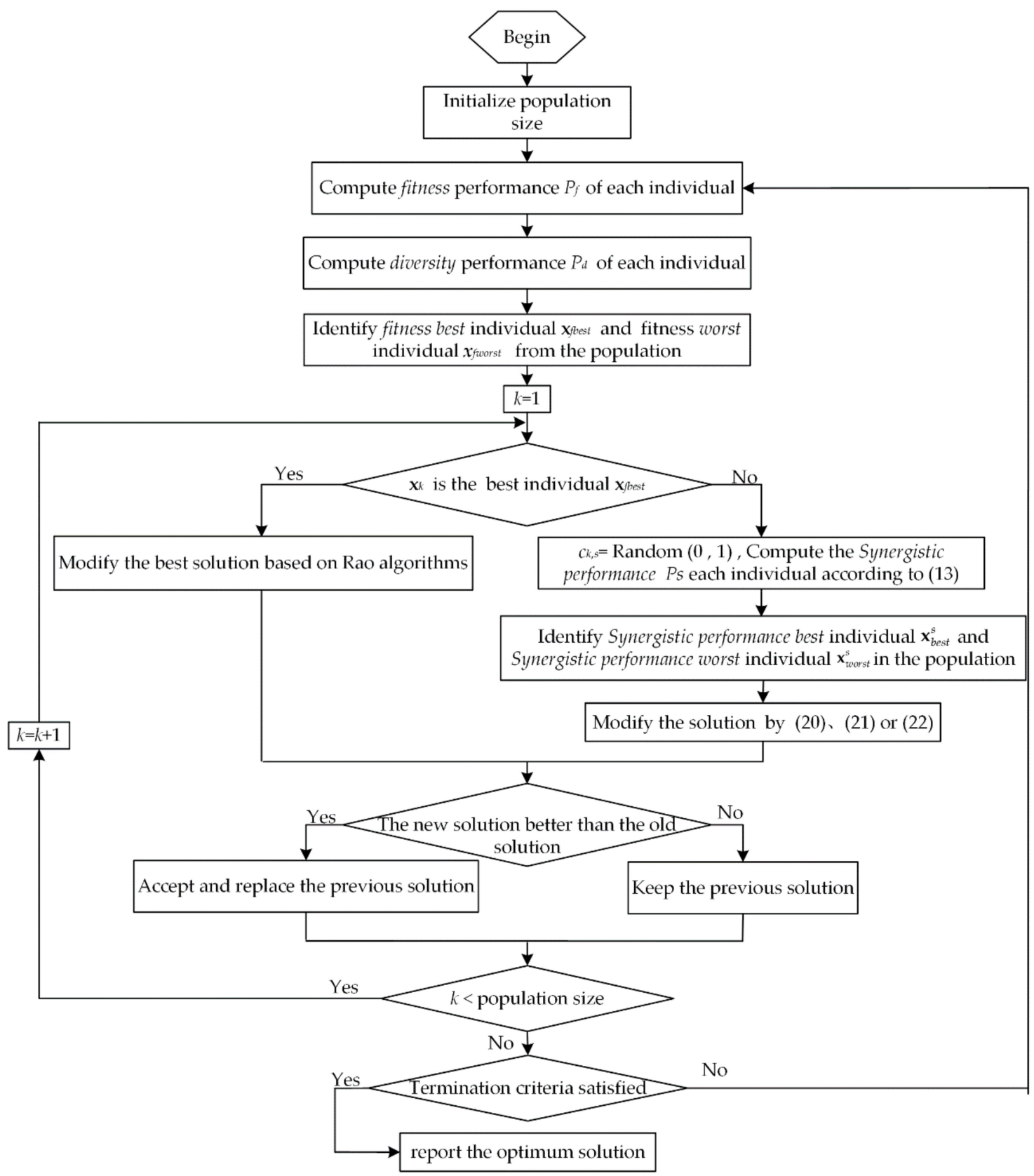

Figure 1. The flow diagram of SP-R. 


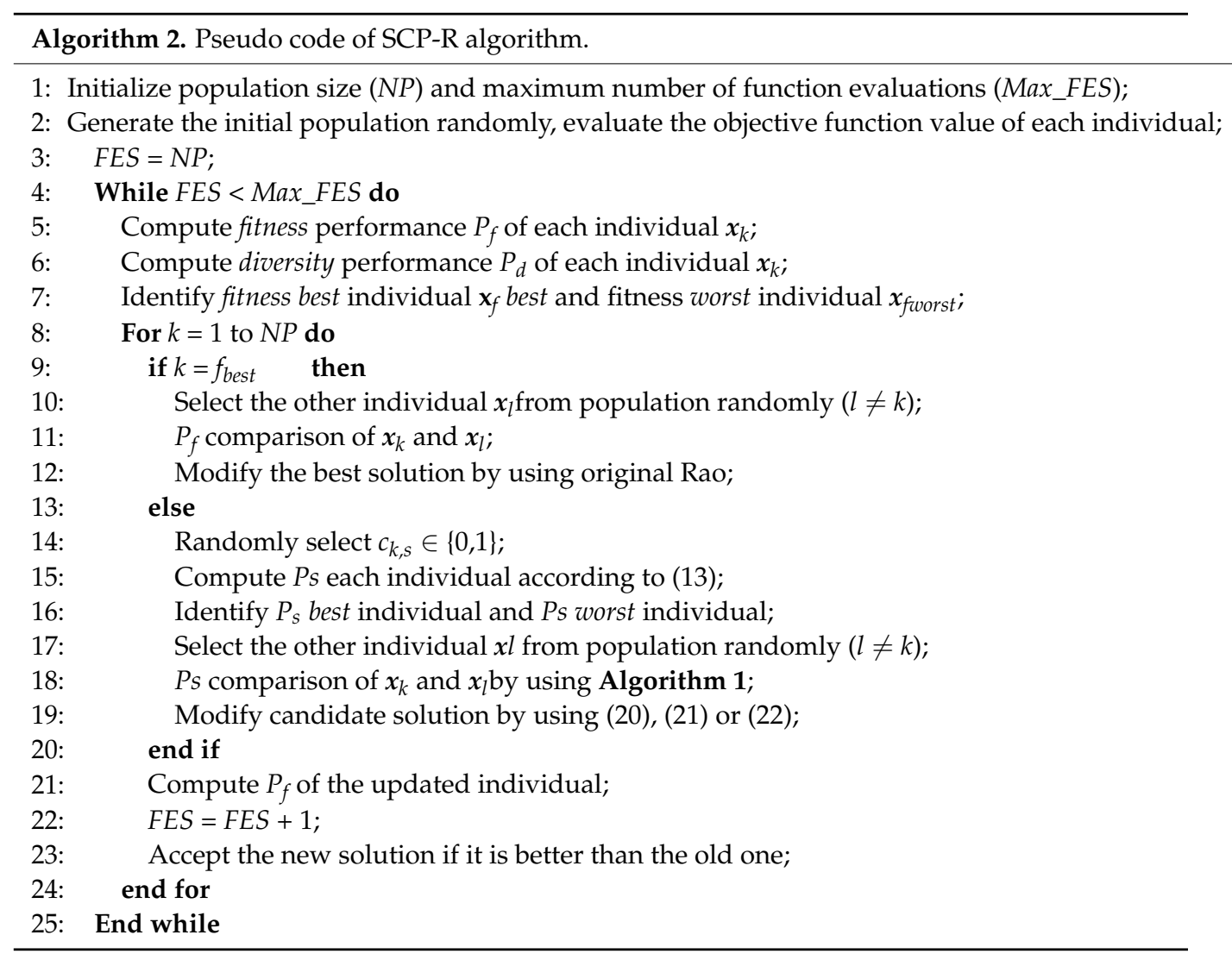

SCP-R algorithms have simple structure and are free from algorithm-specific parameters. During the operation of the algorithm, evolutionary preferences may be dynamically balanced, which is equivalent to the convergence strength being automatically managed. Different strategies are employed to modify the FP optimal candidate and other candidates, which is equivalent to classifying the candidates in the current population. Considering that there are other optimal candidates nearby, a relatively conservative modification strategy is adopted to search the surrounding region. On the contrary, for the other candidates, more radical modification strategies are employed to improve population diversity as well as exploration capabilities.

\section{Experimental Results}

\subsection{Experiment Setup}

To evaluate the performance of the proposed SCP-R algorithms, comparative experiments are carried out with already well established algorithms. These algorithms are the original Rao algorithms [25], Grey Wolf Optimization (GWO) algorithm [28] and Ant Lion Optimization (ALO) algorithm [29]. The proposed model is a dual function model with common parameters. A two-stage experiment is implemented to identify parameters. For the sake of fairness, the parameter configuration of the experiment is the same for different algorithms. The same maximum number of function evaluations (Max_FES) for all algorithms is set to 30,000 in each run. To reduce the statistical error as far as possible, each algorithm is independently conducted 30 times on the problem. The parameter range is fixed in (0.2) to ensure the same search space. In addition, the population size in different stages is set to $N P=20$ and $N P=30$. The reference data used in the parameter identification process is the FEA result of a $2 \mathrm{kw}-\mathrm{SynRM}$ prototype. The design parameters of the prototype are summarized in Table 1. 
Table 1. Parameters of SynRM prototype.

\begin{tabular}{cccc}
\hline Parameters & Values & Parameters & Values \\
\hline Rated Power $(\mathrm{kw})$ & 2 & Rated Voltage $(\mathrm{V})$ & 42 \\
Rated Speed $\left(\mathrm{r} \cdot \mathrm{min}^{-1}\right)$ & 1500 & Rated Current $(\mathrm{A})$ & 35 \\
Rated Torque $(\mathrm{N} \cdot \mathrm{m})$ & 12.7 & Number of pole pairs & 2 \\
Stator Resistance $(\Omega)$ & 0.3 & Moment of inertia $\left(\mathrm{kg} \cdot \mathrm{m}^{-2}\right)$ & 0.025 \\
\hline
\end{tabular}

\subsection{Main Results}

\subsubsection{Results on $q$-Axis Flux Linkage Model}

For the $q$-axis flux linkage model, the different algorithms are independently implemented 30 times to obtain its self-saturation and cross-saturation parameters. Because the exact values of the model parameters are unknown, RMSE $q$ is taken as the standard of accuracy evaluation. The comparison of the best parameter values and the best RMSE among the 30 identification results of different algorithms is given in Table 2. Among them, the best RMSE $q$ are marked in bold. It can be seen from Table 2 that SCP-R algorithms achieve better accuracy than other algorithms, and SCP-R1, SCP-R2 and SCP-R3 algorithm provide the best RMSE $q$ value $\left(2.2243 \times 10^{-4}\right)$. Although the RMSE $q$ of some algorithms is very close to the results obtained by SCP-R algorithms, a smaller RMSE $q$ is conducive to determine the true parameters of the model.

Table 2. Comparison among different algorithms on $q$-axis flux linkage model.

\begin{tabular}{|c|c|c|c|c|c|c|c|c|c|c|}
\hline \multirow{2}{*}{ Algorithm } & \multicolumn{4}{|c|}{ Self-Saturation Coefficients } & \multicolumn{5}{|c|}{ Cross-Saturation Coefficients } & \multirow{2}{*}{$\mathrm{RMSE} q$} \\
\hline & $\alpha_{q}$ & $\beta_{q}$ & $\gamma_{q}$ & $\gamma_{q 1}$ & $k$ & $W$ & $V$ & $V_{1}$ & $\kappa_{q}$ & \\
\hline SCP-R1 & 0.0072 & 1.0324 & 0.1233 & $7.8240 \times 10^{-4}$ & 0.0216 & 0.0020 & 0.0114 & 0.2633 & 0.0758 & $2.2243 \times 10^{-4}$ \\
\hline SCP-R2 & 0.0072 & 1.0324 & 0.1233 & $7.8240 \times 10^{-4}$ & 0.0216 & 0.0020 & 0.0114 & 0.2633 & 0.0758 & $2.2243 \times 10^{-4}$ \\
\hline SCP-R3 & 0.0072 & 1.0324 & 0.1233 & $7.8240 \times 10^{-4}$ & 0.0216 & 0.0020 & 0.0114 & 0.2633 & 0.0758 & $2.2243 \times 10^{-4}$ \\
\hline Rao-1 & 0.0071 & 1.0297 & 0.1238 & $7.8393 \times 10^{-4}$ & 0.0215 & 0.0020 & 0.0115 & 0.2633 & 0.0751 & $2.2443 \times 10^{-4}$ \\
\hline Rao-2 & 0.0079 & 0.2632 & 0.6453 & $7.0144 \times 10^{-4}$ & 0.0215 & 0.0020 & 0.0115 & 0.2633 & 0.0751 & $5.8524 \times 10^{-4}$ \\
\hline Rao-3 & 0.0072 & 1.6691 & 0.0597 & $7.6864 \times 10^{-4}$ & 0.0042 & 0.0016 & 0 & 2.0000 & 2.0000 & $7.0207 \times 10^{-4}$ \\
\hline GWO & 0.0071 & 1.0610 & 0.1251 & $7.8437 \times 10^{-4}$ & 0.0198 & 0.0021 & 0.0112 & 0.2685 & 0.1083 & $2.3034 \times 10^{-4}$ \\
\hline ALO & 0.0140 & 0.0014 & 0.3133 & $7.7888 \times 10^{-4}$ & 0.0197 & 0.0033 & 0.0109 & 0.2151 & 0.0041 & $6.9356 \times 10^{-4}$ \\
\hline
\end{tabular}

\subsubsection{Results on $d$-Axis Flux Linkage Model}

When $d$-axis model parameters are extracted, the best cross-saturation coefficients are applied to the identification of the parameters of the model. Thus, there are only four parameters to be identified. The best estimated parameters and the best RMSEd of different algorithms are listed in Table 3. The SCP-R algorithms also provide the best RMSEd value $\left(4.0815 \times 10^{-4}\right)$ among all compared algorithms.

To illustrate the excellent results of the identification results, the absolute error rate (AER) between the estimated value and the FEA value is shown in Figure 2. Obviously, the estimated value of flux linkages in the entire current range is highly consistent with its FEA value. In terms of AER, except for the very few points on the edge of the model where the AER is close to $4 \%$, the AER at other contrast points does not exceed $1 \%$. 
Table 3. Comparison among different algorithms on $d$-axis flux linkage model.

\begin{tabular}{|c|c|c|c|c|c|}
\hline \multirow{2}{*}{ Algorithm } & \multicolumn{4}{|c|}{ Self-Saturation Coefficients } & \multirow{2}{*}{ RMSE $d$} \\
\hline & $\alpha_{d}$ & $\beta_{d}$ & $\gamma_{d}$ & $\gamma_{d 1}$ & \\
\hline SCP-R1 & 0.0615 & 0.1498 & 0.0100 & 0.0013 & $4.0815 \times 10^{-4}$ \\
\hline SCP-R2 & 0.0615 & 0.1498 & 0.0100 & 0.0013 & $4.0815 \times 10^{-4}$ \\
\hline SCP-R3 & 0.0615 & 0.1498 & 0.0100 & 0.0013 & $4.0815 \times 10^{-4}$ \\
\hline Rao-1 & 0.0615 & 0.1500 & 0.00995 & 0.0013 & $4.0866 \times 10^{-4}$ \\
\hline Rao-2 & 0.0616 & 0.1497 & 0.0100 & 0.0013 & $4.0821 \times 10^{-4}$ \\
\hline Rao-3 & 0.0615 & 0.1498 & 0.0100 & 0.0013 & $4.0817 \times 10^{-4}$ \\
\hline GWO & 0.0615 & 0.1498 & 0.0100 & 0.0013 & $4.0818 \times 10^{-4}$ \\
\hline ALO & 0.0623 & 0.1484 & 0.0098 & 0.0013 & $4.2398 \times 10^{-4}$ \\
\hline
\end{tabular}

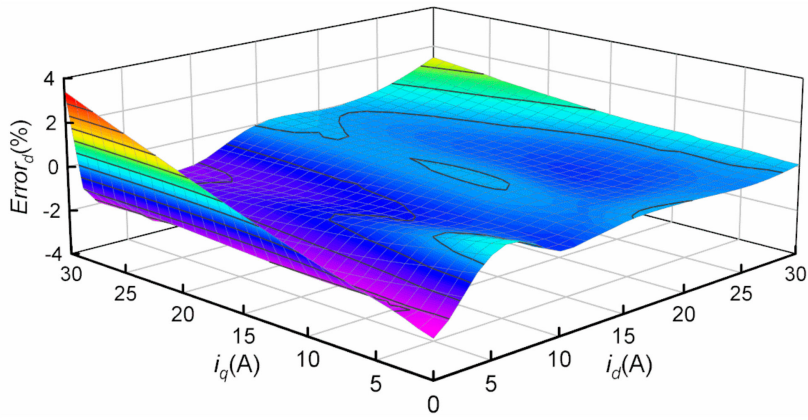

(a)

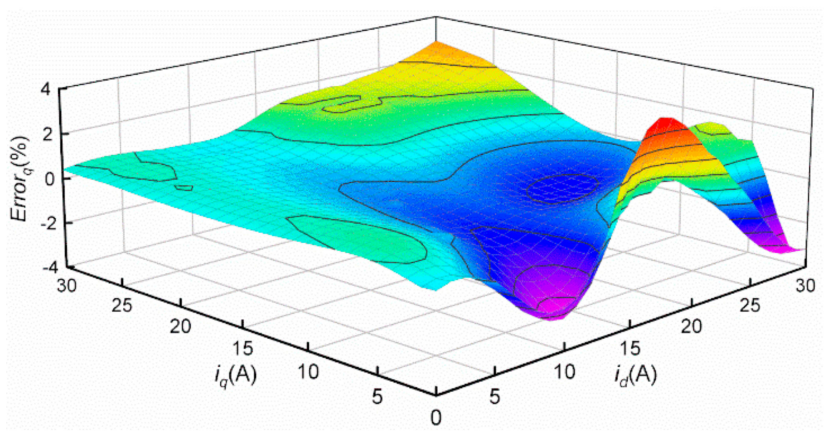

(b)

Figure 2. (a) Mismatch of $d$-axis flux linkage, estimated value vs finite element analysis (FEA) value; (b) Mismatch of $q$-axis flux linkage, estimated value vs FEA value.

\subsection{Results Discussion}

\subsubsection{Robustness of SCP-R Algorithms}

To further evaluate the robustness of the proposed algorithms. Analysis based on RMSE statistics is implemented. The statistical results of the parameter identification of the dual function model by the different algorithms that run 30 times independently are given in Table 4. The results of the algorithms involved are calculated according to the best RMSE (B), worst RMSE (W), average RMSE (M), and standard deviation RMSE (SD). For each model, the best result in the corresponding algorithm is indicated in bold.

In terms of average accuracy and robustness, it can be observed from Table 4 that the overall performance of the proposed SCP-R algorithms is better than other algorithms. For the $q$-axis flux linkage model, the best $\mathrm{M}\left(2.5142 \times 10^{-4}\right)$ and best SD $\left(8.2501 \times 10^{-4}\right)$ are provided by the proposed SCP-R1 algorithm. Specifically, the SCP-R3 algorithm with excellent SD $\left(3.7124 \times 10^{-19}\right)$ is far more robust than other algorithms. The distribution characteristics of the results of 30 independent runs of the different algorithms are presented in Figure 3 using box plots. The distribution range of the parameter identification results also demonstrates the superiority of the proposed SCP-R algorithms. Similarly, the results distribution corresponding to the SCP-R3 algorithm is the most concentrated. Obviously, the proposed SCP-R has better performance on searching accuracy and reliability for solving the parameters identification problems of the SynRM magnetic model, and its performance is competitive in contrast with all compared algorithms. 
Table 4. Statistical results of root mean square (RMSE) of different algorithms for Synchronous Reluctance Motor (SynRM) magnetic model (30,000 function evaluations).

\begin{tabular}{|c|c|c|c|c|c|c|}
\hline \multirow{2}{*}{ Model } & \multirow{2}{*}{ Population Size } & \multirow{2}{*}{ Algorithm } & \multicolumn{4}{|c|}{ RMSE } \\
\hline & & & B & $\mathbf{M}$ & $\mathbf{W}$ & SD \\
\hline \multirow{8}{*}{$q$-axis flux linkage model } & \multirow{8}{*}{$N P=20$} & SCP-R1 & $2.2243 \times 10^{-4}$ & $2.5142 \times 10^{-4}$ & $5.6827 \times 10^{-4}$ & $8.2501 \times 10^{-4}$ \\
\hline & & SCP-R2 & $2.2243 \times 10^{-4}$ & $3.2947 \times 10^{-4}$ & 0.0022 & $3.7615 \times 10^{-4}$ \\
\hline & & SCP-R3 & $2.2243 \times 10^{-4}$ & $2.7336 \times 10^{-4}$ & 0.0011 & $1.7291 \times 10^{-4}$ \\
\hline & & Rao-1 & $2.2243 \times 10^{-4}$ & 0.0017 & 0.0238 & 0.0043 \\
\hline & & Rao-2 & $5.8524 \times 10^{-4}$ & 0.0053 & 0.0238 & 0.0074 \\
\hline & & Rao-3 & $7.0207 \times 10^{-4}$ & 0.0073 & 0.0238 & 0.0093 \\
\hline & & GWO & $2.3034 \times 10^{-4}$ & 0.0018 & 0.0050 & 0.0012 \\
\hline & & ALO & $6.9356 \times 10^{-4}$ & 0.0022 & 0.0049 & 0.0010 \\
\hline \multirow{8}{*}{$d$-axis flux linkage model } & \multirow{8}{*}{$N P=30$} & SCP-R1 & $4.0815 \times 10^{-4}$ & $4.0815 \times 10^{-4}$ & $4.0815 \times 10^{-4}$ & $6.4849 \times 10^{-12}$ \\
\hline & & SCP-R2 & $4.0815 \times 10^{-4}$ & $4.3181 \times 10^{-4}$ & $8.7509 \times 10^{-4}$ & $6.2187 \times 10^{-10}$ \\
\hline & & SCP-R3 & $4.0815 \times 10^{-4}$ & $4.0815 \times 10^{-4}$ & $4.0815 \times 10^{-4}$ & $3.7124 \times 10^{-19}$ \\
\hline & & Rao-1 & $4.0866 \times 10^{-4}$ & 0.0010 & 0.0034 & 0.0014 \\
\hline & & Rao-2 & $4.0821 \times 10^{-4}$ & 0.0028 & 0.0232 & 0.0041 \\
\hline & & Rao-3 & $4.0817 \times 10^{-4}$ & 0.0023 & 0.0034 & 0.0012 \\
\hline & & GWO & $4.0818 \times 10^{-4}$ & 0.0029 & 0.0232 & 0.0056 \\
\hline & & ALO & $4.2398 \times 10^{-4}$ & 0.0032 & 0.0180 & 0.0017 \\
\hline
\end{tabular}

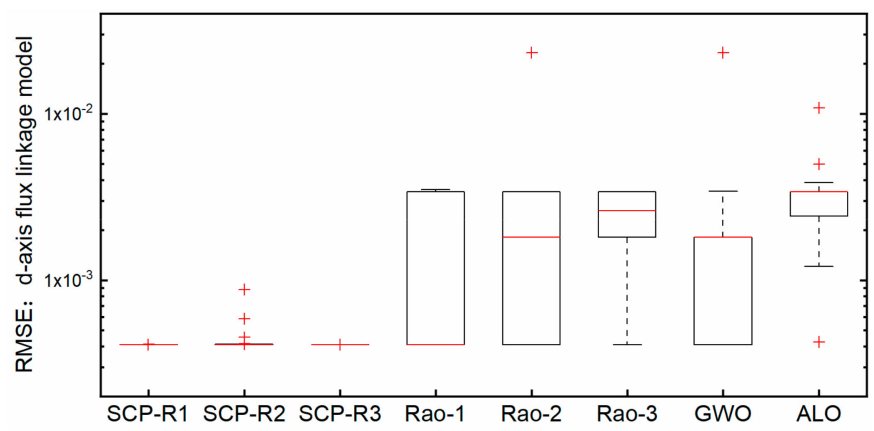

(a)

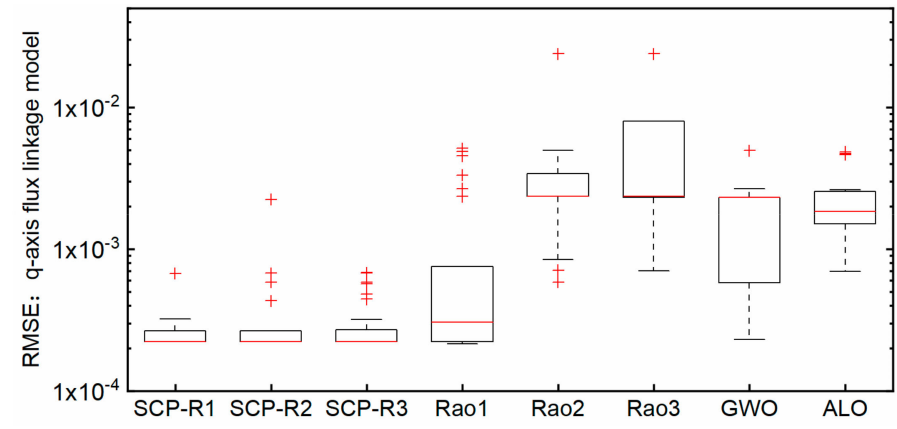

(b)

Figure 3. Best RMSE boxplot over 30 runs of different algorithms for SynRM magnetic model (a) Statistical results of RMSEd (b) Statistical results of RMSEq.

\subsubsection{Discussion on Optimal Solution Search Path Update Mechanism of SCP-R}

In order to demonstrate the effectiveness of the proposed SCP-R framework, comparative experiments for two variants of SCP-R algorithms are implemented in this subsection. The algorithms are obtained by modifying the optimal search path mechanism of the SCP-R algorithms. For the mechanism of the SCP-R2 algorithm, the interaction between the current candidate and the randomly selected candidate does not depend on SCP (denoted as M-SCP-R2). Similarly, the modification to the SCP-R3 algorithm is denoted as M-SCP-R3. Note that the SCP independent SCP-R1 algorithm will degenerate to the original Rao-1. The comparison of these two algorithms has been implemented in the previous subsections. The statistical results of the two variants are presented in Table 5. For each model, the values shown in bold in Table 5 indicate the comparatively better results of the respective algorithms. In terms of average accuracy and robustness, it can be clearly observed from Table 5 that the overall performance of the proposed SCP-R algorithms is better than the other variants. The obvious difference between the results of the SCP-R algorithm and the corresponding variants indicates that the SCP-R framework is beneficial to optimize the original optimal solution update path mechanism. In summary, the excellent performance 
of the SCP-R algorithms verifies that the SCP-R-framework-based optimal solution search path update has achieved appropriate balance between exploration and exploitation.

Table 5. Statistical results of RMSE of different SCP-R algorithms for SynRM magnetic model (30,000 function evaluations).

\begin{tabular}{|c|c|c|c|c|c|c|}
\hline \multirow{2}{*}{ Model } & \multirow{2}{*}{ Population Size } & \multirow{2}{*}{ Algorithm } & \multicolumn{4}{|c|}{ RMSE } \\
\hline & & & B & $\mathbf{M}$ & $\mathbf{W}$ & SD \\
\hline \multirow{4}{*}{$\begin{array}{l}q \text {-axis flux linkage } \\
\text { model }\end{array}$} & \multirow{4}{*}{$N P=20$} & SCP-R2 & $2.2243 \times 10^{-4}$ & $3.2947 \times 10^{-4}$ & 0.0022 & $3.7615 \times 10^{-4}$ \\
\hline & & M-SCP-R2 & $2.2243 \times 10^{-4}$ & 0.0097 & 0.2617 & 0.0476 \\
\hline & & SCP-R3 & $2.2243 \times 10^{-4}$ & $2.7336 \times 10^{-4}$ & 0.0011 & $1.7291 \times 10^{-4}$ \\
\hline & & M-SCP-R3 & $2.2243 \times 10^{-4}$ & 0.0034 & 0.0722 & 0.0131 \\
\hline \multirow{4}{*}{$\begin{array}{l}d \text {-axis flux linkage } \\
\text { model }\end{array}$} & \multirow{4}{*}{$N P=30$} & SCP-R2 & $4.0815 \times 10^{-4}$ & $4.3181 \times 10^{-4}$ & $8.7509 \times 10^{-4}$ & $6.2187 \times 10^{-10}$ \\
\hline & & M-SCP-R2 & 0.0111 & 0.0112 & 0.0113 & $8.0570 \times 10^{-5}$ \\
\hline & & SCP-R3 & $4.0815 \times 10^{-4}$ & $4.0815 \times 10^{-4}$ & $4.0815 \times 10^{-4}$ & $3.7124 \times 10^{-19}$ \\
\hline & & M-SCP-R3 & 0.0111 & 0.0112 & 0.0113 & $6.6143 \times 10^{-5}$ \\
\hline
\end{tabular}

\section{Conclusions}

In this paper, SCP-R algorithms for the SynRM magnetic model parameter identification are proposed, which focuses on establishing a quantitative method for overall performance of candidates in current population. By implementing the SCP-based quantification framework, fitness and diversity information of candidates are uniformly employed for evolutionary guidance. A good balance between exploration and exploitation can be achieved. Experiment results further demonstrated that SCP-R algorithms perform better in terms of accuracy and reliability when compared with other well-established algorithms. Therefore, combining the SCP-based contribution measurement together with the proposed SCP-R framework may further improve the SynRM magnetic model parameter identification performance.

Author Contributions: Conceptualization, L.R. and G.L., Data curation, L.R. and Y.Z.; Formal analysis, L.R., Y.Z. and Z.L., Funding acquisition, G.L. and Z.L.; Investigation, Y.Z. and Z.L.; Methodology, L.R., G.L. and Z.L.; Project administration, G.L. and Z.L.; Resources, G.L.; Software, L.R.; Supervision, G.L.; Validation, L.R., Y.Z. and Z.L.; Visualization, L.R. and Y.Z.; Writing—original draft, L.R.; Writing-review \& editing, L.R. and Y.Z. All authors have read and agreed to the published version of the manuscript.

Funding: This research received no external funding.

Acknowledgments: The authors would like to thank the reviewers for their helpful suggestions. This work is supported by the project of Science and Technology Commission Shanghai Municipality (No. 17511102302).

Conflicts of Interest: The authors declare no conflict of interest.

\section{References}

1. Ozcelik, N.G.; Dogru, U.E.; Imeryuz, M.; Ergene, L.T. Synchronous Reluctance Motor vs. Induction Motor at Low-Power Industrial Applications: Design and Comparison. Energies 2019, 12, 2190. [CrossRef]

2. Yang, Z.; Shang, F.; Brown, I.P.; Krishnamurthy, M. Comparative study of interior permanent magnet, induction, and switched reluctance motor drives for EV and HEV applications. IEEE Trans. Transp. Electrif. 2015, 1, 245-254. [CrossRef]

3. Song, F.; Zhou, Y.; Chang, L.; Zhang, H. Modeling Space-Terrestrial Integrated Networks with Smart Collaborative Theory. IEEE Netw. 2019, 33, 51-57. [CrossRef]

4. Wiedemann, S.; Hall, S.; Kennel, R.M.; Alaküla, M. Dynamic testing characterization of a synchronous reluctance machine. IEEE Trans. Ind. Appl. 2017, 54, 1370-1378.

5. Qu, Z.; Tuovinen, T.; Hinkkanen, M. Inclusion of magnetic saturation in dynamic models of synchronous reluctance motors. In Proceedings of the 20th International Conference on Electrical Machines (ICEM), Marseille, France, 2-5 September 2012; pp. 994-1000.

6. Song, F.; Ai, Z.; Zhang, H.; You, I.; Li, S. Smart Collaborative Balancing for Dependable Network Components in Cyber-Physical Systems. IEEE Trans. Ind. Inform. 2020. [CrossRef] 
7. Ortombina, L.; Tinazzi, F.; Zigliotto, M. Magnetic Modeling of Synchronous Reluctance and Internal Permanent Magnet Motors Using Radial Basis Function Networks. IEEE Trans. Ind. Electron. 2017, 65, 1140-1148. [CrossRef]

8. Sun, Y.; Xu, J.; Lin, G.; Ji, W.; Wang, L. RBF Neural Network-Based Supervisor Control for Maglev Vehicles on an Elastic Track with Network Time-Delay. IEEE Trans. Ind. Inform. 2020. [CrossRef]

9. Sun, Y.; Xu, J.; Wu, H.; Lin, G.; Mumtaz, S. Deep Learning Based Semi-Supervised Control for Vertical Security of Maglev Vehicle with Guaranteed Bounded Airgap. IEEE Trans. Intell. Transp. Syst. 2021. [CrossRef]

10. Hinkkanen, M.; Pescetto, P.; Molsa, E. Sensorless self-commissioning of synchronous reluctance motors at standstill. In Proceedings of the XXII International Conference on Electrical Machines (ICEM), Lausanne, Switzerland, 4-7 September 2016.

11. Niu, Q.; Zhang, L.; Li, K. A biogeography-based optimization algorithm with mutation strategies for model parameter estimation of solar and fuel cells. Energy Convers. Manag. 2014, 86, 1173-1185. [CrossRef]

12. Accetta, A.; Cirrincione, M.; Pucci, M.; Sferlazza, A. A saturation model of the synchronous reluctance motor and its identification by genetic algorithms. In Proceedings of the 2018 IEEE Energy Conversion Congress and Exposition (ECCE), Portland, OR, USA, 23-27 September 2018; pp. 4460-4465.

13. Qiu, Y.; Kang, Q.; Wang, L.; Wu, Q. A parameter optimization method for dq axis inductance model of synchronous reluctance motors considering cross-coupling magnetic saturation. Trans. China Electrotech. Soc. 2017, 32, 85-92.

14. Huynh, D.C.; Dunnigan, M.W. Parameter estimation of an induction machine using advanced particle swarm optimisation algorithms. IET Electr. Power Appl. 2010, 4, 748-760. [CrossRef]

15. Wang, C.; Liu, Y.; Liang, X.; Guo, H.; Chen, Y.; Zhao, Y. Self-adaptive differential evolution algorithm with hybrid mutation operator for parameters identification of PMSM. Soft Comput. 2018, 22, 1263-1285. [CrossRef]

16. Kim, J.W.; Kim, S.W. Parameter identification of induction motors using dynamic encoding algorithm for searches. IEEE Trans. Energy Convers. 2005, 20, 16-24. [CrossRef]

17. Liu, Z.H.; Li, X.H.; Wu, L.H.; Zhou, S.W.; Liu, K. GPU-accelerated parallel coevolutionary algorithm for parameters identification and temperature monitoring in permanent magnet synchronous machines. IEEE Trans. Ind. Inform. 2015, 11, 1220-1230. [CrossRef]

18. Juang, C.F. A hybrid of genetic algorithm and particle swarm optimization for recurrent network design. IEEE Trans. Syst. Man Cybern. 2004, 34, 997-1006. [CrossRef]

19. Song, F.; Zhou, Y.; Wang, Y.; Zhao, T.; You, I.; Zhang, H. Smart Collaborative Distribution for Privacy Enhancement in Moving Target Defense. Inf. Sci. 2019, 479, 593-606.

20. Yu, K.; Qu, B.; Yue, C. A performance-guided JAYA algorithm for parameters identification of photovoltaic cell and module. Appl. Energy 2019, 237, 241-257. [CrossRef]

21. Gong, W.; Cai, Z. Differential evolution with ranking-based mutation operators. IEEE Trans. Cybern. 2013, 43, 2066-2081. [CrossRef] [PubMed]

22. Wang, J.; Liao, J.; Zhou, Y.; Cai, Y. Differential Evolution Enhanced with Multi-objective Sorting-Based Mutation Operators. IEEE Trans. Cybern. 2017, 44, 2792-2805. [CrossRef]

23. Song, F.; Zhu, M.; Zhou, Y.; You, I.; Zhang, H. Smart Collaborative Tracking for Ubiquitous Power IoT in Edge-Cloud Interplay Domain. IEEE Internet Things J. 2020, 7, 6046-6055. [CrossRef]

24. Maldonado, V.R. Synchronous Reluctance Motor and Drive Package: The Energy Efficiency Alternative. 2013. Available online: http:/ / www.abb.com (accessed on 2 March 2021).

25. Rao, R. Rao algorithms: Three metaphor-less simple algorithms for solving optimization problems. Int. J. Ind. Eng. Comput. 2020, 11,107-130. [CrossRef]

26. Corriveau, G.; Guilbault, R.; Tahan, A.; Sabourin, R. Review and study of genotypic diversity measures for real-coded representations. IEEE Trans. Evol. Comput. 2012, 16, 695-710. [CrossRef]

27. Song, F.; Ai, Z.; Zhou, Y.; You, I.; Choo, R.; Zhang, H. Smart Collaborative Automation for Receive Buffer Control in Multipath Industrial Networks. IEEE Trans. Ind. Inform. 2020, 16, 1385-1394. [CrossRef]

28. Mirjalili, S.; Mirjalili, S.M.; Lewis, A. Grey wolf optimizer. Adv. Eng. Softw. 2014, 69, 46-61. [CrossRef]

29. Mirjalili, S. The ant lion optimizer. Adv. Eng. Softw. 2015, 83, 80-98. [CrossRef] 